NASA Technical Memorandum 105693

\title{
Numerical Solution of a Three-Dimensional Cubic Cavity Flow by Using the Boltzmann Equation
}

Danny P. Hwang

Lewis Research Center

Cleveland, Ohio

Prepared for the

18th International Symposium on Rarefield Gas Dynamics sponsored by the University of British Columbia

Vancouver, Canada, July 26-31, 1992

\section{N/SA}




\title{
NUMERICAL SOLUTION OF A THREE-DIMENSIONAL CUBIC
}

\section{CAVITY FLOW BY USING THE BOLTZMANN EQUATION}

\author{
Danny P. Hwang* \\ National Aeronautics and Space Administration \\ Lewis Research Center \\ Cleveland, Ohio 44135
}

\section{Summary}

A three-dimensional cubic cavity flow has been analyzed for diatomic gases by using the Boltzmann equation with the Bhatnagar-Gross-Krook (B-G-K) model. The method of discrete ordinate was applied, and the diffuse reflection boundary condition was assumed. The results, which show a consistent trend toward the Navier-Stokes solution as the Knudson number is reduced, give us confidence to apply the method to a threedimensional geometry for practical predictions of rarefied-flow characteristics. The CPU time and the main memory required for a threedimensional geometry using this method seem reasonable.

\section{Symbols}

a defined in equations (5) and (6)

$\mathrm{E}_{\sigma} \quad$ energy level (equal to the roots of the Laguerre polynomial of degree $n$ )

F Maxwellian distribution function

f distribution function

imax maximum $i$ index

jmax $\quad$ maximum $\mathrm{j}$ index

Kn Knudson number

k Boltzmann constant

$\mathrm{kmax} \quad \operatorname{maximum} \mathrm{k}$ index

\footnotetext{
* Aerospace Engineer.
} 


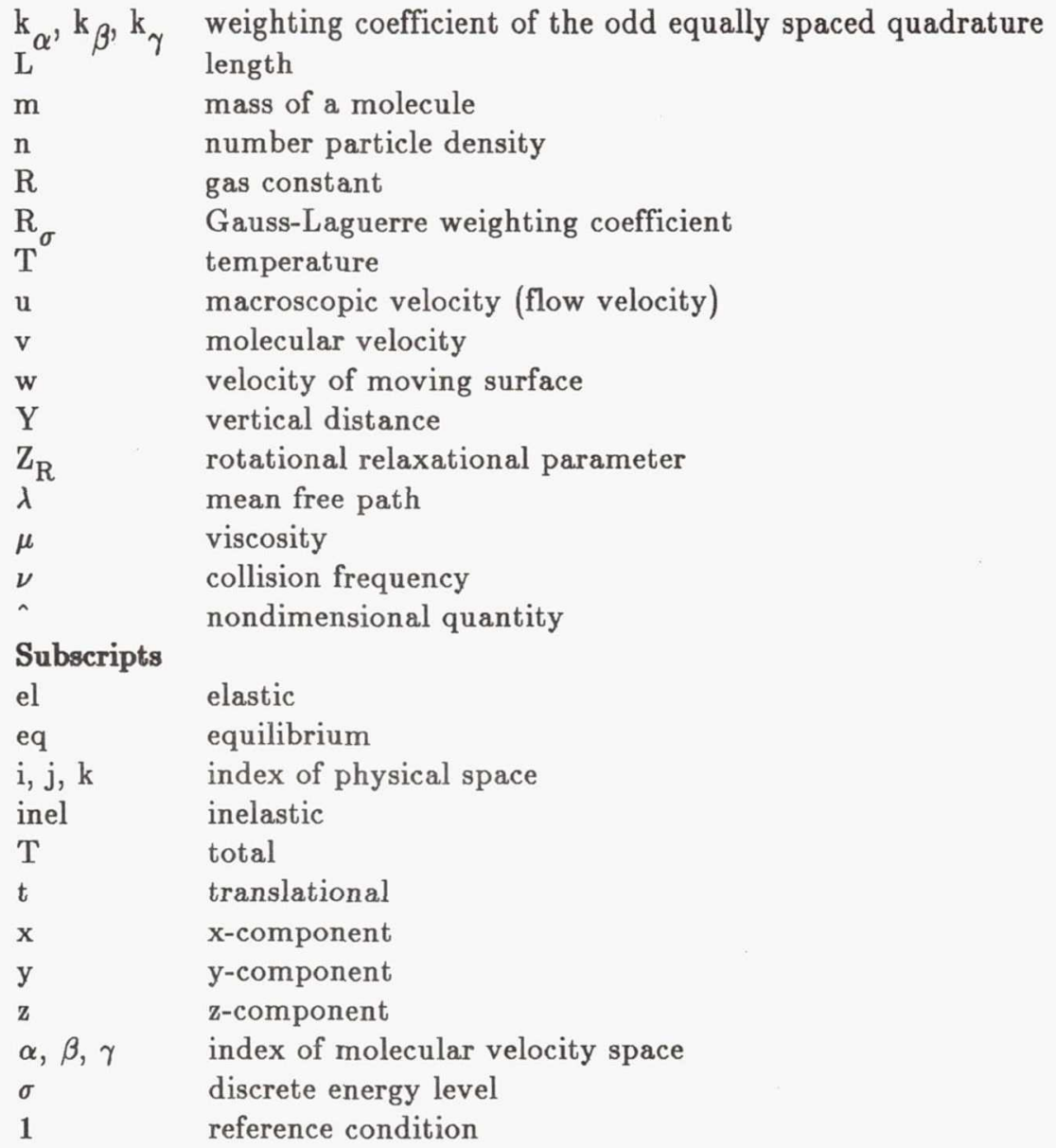

\section{Introduction}

With space vehicles orbiting the world at hypersonic speed, numerical methods to cover all flow regimes become more important. Advances in computer hardware in recent years allow methods based on the kinetic theory to be used for practical applications. Many two-dimensional problems have been solved by the kinetic approach. ${ }^{1-7}$ A limited number of three-dimensional problems have been solved recently by the directsimulation Monte Carlo (DSMC) method. ${ }^{8-10}$ However, there are no threedimensional numerical solutions based on the discrete ordinate method ${ }^{11}$ applied to a Boltzmann equation.

A cubic cavity flow problem has been solved for diatomic gases using the Boltzmann equation with the Bhatnagar-Gross-Krook (B-G-K) model. ${ }^{12,13}$ Because neither theoretical nor experimental data are available for cubic cavity flow in free molecule flow, slip flow, and transition flow regimes, efforts have been made to obtain a continuum flow solution as 
closed as possible so that the results from this study can be compared with the results of the Navier-Stokes solution. ${ }^{14}$

\section{Governing Equation}

The geometry of the problem considered is shown in figure 1. A cubic cavity with the same dimension on all sides is filled with diatomic gas (such as air), and the wall temperature is assumed to be the same on all walls. The top surface $(\mathrm{j}=\mathrm{jmax}$ surface $)$ is moving at a constant speed, and steady-state conditions are reached and analyzed numerically.

The distribution function at the energy level $\mathrm{E}_{\sigma}, \mathrm{f}_{\sigma}$, is governed by the Boltzmann equation with the Bhatnagar-Gross-Krook-Morse model for the collision terms:

$$
\mathrm{v}_{\mathrm{x}} \frac{\partial \mathrm{f}_{\sigma}}{\partial \mathrm{x}}+\mathrm{v}_{\mathrm{y}} \frac{\partial \mathrm{f}_{\sigma}}{\partial \mathrm{y}}+\mathrm{v}_{\mathrm{z}} \frac{\partial \mathrm{f}_{\sigma}}{\partial \mathrm{z}}=\nu_{\mathrm{el}}\left(\mathrm{F}_{\mathrm{t} \sigma}-\mathrm{f}_{\sigma}\right)+\nu_{\mathrm{inel}}\left(\mathrm{F}_{\mathrm{i} \sigma}-\mathrm{f}_{\sigma}\right)
$$

where

$$
\begin{aligned}
& \mathrm{F}_{\mathrm{t} \sigma}=\mathrm{n}_{\sigma}\left(\frac{\mathrm{m}}{2 \pi \mathrm{kT}}\right)^{3 / 2} \mathrm{e}^{-\frac{\mathrm{m}}{2 \mathrm{kT}}\left(\left(\mathrm{v}_{\mathrm{x}}-\mathrm{u}_{\mathrm{x}}\right)^{2}+\left(\mathrm{v}_{\mathrm{y}}-\mathrm{u}_{\mathrm{y}}\right)^{2}+\left(\mathrm{v}_{\mathrm{z}}-\mathrm{u}_{\mathrm{z}}\right)^{2}\right)} \\
& \mathrm{F}_{\mathrm{i} \sigma}=\mathrm{n}_{\sigma \mathrm{eq}}\left(\frac{\mathrm{m}}{2 \pi \mathrm{kT}}\right)^{3 / 2} \mathrm{e}^{-\frac{\mathrm{m}}{2 \mathrm{k} \mathrm{T}_{\mathrm{T}}}\left(\left(\mathrm{v}_{\mathrm{x}}-\mathrm{u}_{\mathrm{x}}\right)^{2}+\left(\mathrm{v}_{\mathrm{y}}-\mathrm{u}_{\mathrm{y}}\right)^{2}+\left(\mathrm{v}_{\mathrm{z}}-\mathrm{u}_{\mathrm{z}}\right)^{2}\right)}
\end{aligned}
$$

The elastic collision frequency $\nu_{\mathrm{el}}^{15,16}$ is given by

$$
\nu_{\mathrm{el}}=\frac{\mathrm{nkT}_{\mathrm{t}}}{(1+\mathrm{a}) \mu}
$$

whereas the inelastic collision frequency $\nu_{\text {inel }}$ is related to $\nu_{\mathrm{el}}$ in the formula of

$$
\begin{gathered}
\nu_{\text {inel }}=\mathrm{a} \nu_{\text {el }} \\
\mathrm{Z}_{\mathrm{R}}=\frac{5(1+\mathrm{a})}{3 \mathrm{a}}
\end{gathered}
$$

where the rotational relaxational parameter $\mathrm{Z}_{\mathrm{R}}$ must be obtained by experiment. The viscosity-temperature relation of the form 


$$
\frac{\mu}{\mu_{1}}=\left(\frac{\mathrm{T}_{\mathrm{t}}}{\mathrm{T}_{1}}\right)^{\mathrm{s}}
$$

is assumed and the value of $\mathrm{s}$ can be found in reference 17; for the current study, a and $\mathrm{s}$ are equal to 0.4 and 0.756 , respectively, for air. The subscript 1 indicates the reference condition. The reference condition used in this study is the equilibrium flow in the cubic cavity when the top surface is stationary.

The reference viscosity is related to the reference mean free path $\lambda_{1}$ by the relation

$$
\mu_{1}=\frac{5}{16} \mathrm{mn}_{1} \lambda_{1}\left(2 \pi \mathrm{RT}_{1}\right)^{1 / 2}
$$

Combining equations (4) to (8) gives

$$
\nu_{\text {el }}=\frac{16}{5(1+\mathrm{a})} \frac{\mathrm{nkT}_{\mathrm{t}}}{\lambda_{1} \mathrm{mn}_{1}\left(2 \pi \mathrm{RT}_{1}\right)^{1 / 2}}\left(\frac{\mathrm{T}_{\mathrm{t}}}{\mathrm{T}_{1}}\right)^{-\mathrm{s}}
$$

All macroscopic quantities, which will be listed in the nondimensional form, can be calculated in terms of $f_{\sigma}$.

\section{Nondimensionalisation}

A characteristic velocity used for nondimensionalization is defined as

$$
\mathrm{V}_{1}=\left(2 \mathrm{RT}_{1}\right)^{1 / 2}
$$

The definitions of nondimensional variables are defined as follows:

$$
\begin{gathered}
\hat{\mathrm{n}}=\frac{\mathrm{n}}{\mathrm{n}_{1}} ; \hat{\mathrm{T}}=\frac{\mathrm{T}}{\mathrm{T}_{1}} \\
\hat{\mathrm{u}}_{\mathrm{x}}=\frac{\mathrm{u}_{\mathrm{x}}}{\mathrm{v}_{1}} ; \hat{\mathrm{u}}_{\mathrm{y}}=\frac{\mathrm{u}_{\mathrm{y}}}{\mathrm{v}_{1}} ; \hat{\mathrm{u}}_{\mathrm{z}}=\frac{\mathrm{u}_{\mathrm{z}}}{\mathrm{v}_{1}} \\
\hat{\mathrm{v}}_{\mathbf{x}}=\frac{\mathrm{v}_{\mathrm{x}}}{\mathrm{v}_{1}} ; \hat{\mathrm{v}}_{\mathbf{y}}=\frac{\mathrm{v}_{\mathbf{y}}}{\mathrm{v}_{1}} ; \hat{\mathrm{v}}_{\mathrm{z}}=\frac{\mathrm{v}_{\mathbf{z}}}{\mathrm{v}_{1}}
\end{gathered}
$$




$$
\begin{gathered}
\hat{\mathrm{E}}_{\sigma}=\frac{\mathrm{E}_{\sigma}}{\mathrm{kT}_{1}} \\
\hat{\mathrm{x}}=\frac{\mathrm{x}}{\mathrm{L}_{1}} ; \hat{\mathrm{y}}=\frac{\mathrm{y}}{\mathrm{L}_{1}} ; \hat{\mathrm{z}}=\frac{\mathrm{z}}{\mathrm{L}_{1}} \\
\hat{\nu}_{\mathrm{el}}=\frac{\nu_{\mathrm{el}} \mathrm{L}_{1}}{\mathrm{~V}_{1}} \\
\hat{\mathrm{f}}_{\sigma}=\frac{\mathrm{f}_{\sigma} \mathrm{V}_{1}^{3}}{\mathrm{n}_{1}} ; \hat{\mathrm{F}}_{\mathrm{t} \sigma}=\frac{\mathrm{F}_{\mathrm{t} \sigma} \mathrm{V}_{1}^{3}}{\mathrm{n}_{1}} ; \hat{\mathrm{F}}_{\mathrm{i} \sigma}=\frac{\mathrm{F}_{\mathrm{i} \sigma} \mathrm{V}_{1}^{3}}{\mathrm{n}_{1}}
\end{gathered}
$$

Equation (1) becomes

$$
\hat{\mathrm{v}}_{\mathbf{x}} \frac{\partial \hat{\mathrm{f}}_{\sigma}}{\partial \hat{\mathrm{x}}}+\hat{\mathrm{v}}_{\mathbf{y}} \frac{\partial \hat{\mathrm{f}}_{\sigma}}{\partial \hat{\mathrm{y}}}+\hat{\mathrm{v}}_{\mathbf{z}} \frac{\partial \hat{\mathrm{f}}_{\sigma}}{\partial \hat{\mathrm{z}}}=\hat{\nu}_{\mathrm{el}}\left(\left(\hat{\mathrm{F}}_{\mathrm{t} \sigma}-\hat{\mathrm{f}}_{\sigma}\right)+\mathrm{a}\left(\hat{\mathrm{F}}_{\mathrm{i} \sigma}-\hat{\mathrm{f}}_{\sigma}\right)\right)
$$

where

$$
\begin{gathered}
\hat{\mathrm{F}}_{\mathrm{t} \sigma}=\hat{\mathrm{n}}_{\sigma}\left(\frac{1}{\pi \hat{\mathrm{T}}_{\mathrm{t}}}\right)^{3 / 2} \mathrm{e}^{-\frac{\left(\hat{\mathrm{v}}_{\mathrm{x}}-\hat{\mathrm{u}}_{\mathrm{x}}\right)^{2}+\left(\hat{\mathrm{v}}_{\mathrm{y}}-\hat{\mathrm{u}}_{\mathrm{y}}\right)^{2}+\left(\hat{\mathrm{v}}_{\mathrm{z}}-\hat{\mathrm{u}}_{\mathrm{z}}\right)^{2}}{\hat{\mathrm{T}}_{\mathrm{t}}}} \\
\hat{\mathrm{F}}_{\mathrm{i} \sigma}=\hat{\mathrm{n}}_{\sigma \mathrm{eq}}\left(\frac{1}{\pi \hat{\mathrm{T}}_{\mathrm{T}}}\right)^{3 / 2} \mathrm{e}^{-\frac{\left(\hat{\mathrm{v}}_{\mathrm{x}}-\hat{\mathrm{u}}_{\mathrm{x}}\right)^{2}+\left(\hat{\mathrm{v}}_{\mathrm{y}}-\hat{\mathrm{u}}_{\mathrm{y}}\right)^{2}+\left(\hat{\mathrm{v}}_{\mathrm{z}}-\hat{\mathrm{u}}_{\mathrm{z}}\right)^{2}}{\hat{\mathrm{T}}_{\mathrm{T}}}} \\
\hat{\nu}_{\mathrm{el}}=\frac{8 \hat{\mathrm{n}}}{5(1+\mathrm{a}) \sqrt{\pi \mathrm{Kn}}} \hat{\mathrm{T}}_{\mathrm{t}}^{1-8} \\
\mathrm{Kn}=\frac{\lambda_{1}}{\mathrm{~L}}
\end{gathered}
$$




$$
\begin{aligned}
& \hat{\mathbf{n}}_{\sigma}=\int_{-\infty}^{+\infty} \int_{-\infty}^{+\infty} \int_{-\infty}^{+\infty} \hat{\mathrm{f}}_{\sigma} \mathrm{d} \hat{\mathrm{v}}_{\mathbf{x}} \mathrm{d} \hat{\mathrm{v}}_{\mathbf{y}} \mathrm{d} \hat{\mathrm{v}}_{\mathbf{z}} \\
& \hat{\mathbf{n}}=\sum_{\sigma} \hat{\mathbf{n}}_{\sigma} \\
& \hat{\mathrm{n}}_{\sigma \mathrm{eq}}=\hat{\mathrm{n}}\left\{\frac{\exp \left(-\frac{\hat{\mathrm{E}}_{\sigma}}{\hat{\mathrm{T}}_{\mathrm{T}}}\right)}{\sum_{\mathrm{s}} \exp \left(-\frac{\hat{\mathrm{E}}_{\mathrm{s}}}{\hat{\mathrm{T}}_{\mathrm{T}}}\right)}\right\}=\left(\frac{\hat{\mathrm{n}}}{\hat{\mathrm{T}}_{\mathrm{T}}}\right) \mathrm{R}_{\sigma} \exp \left[\hat{\mathrm{E}}_{\sigma}\left(1-\frac{1}{\hat{\mathrm{T}}_{\mathrm{T}}}\right)\right] \text { (from ref. 1) } \\
& \hat{\mathrm{u}}_{\mathrm{x}}=\frac{1}{\hat{\mathrm{n}}} \sum_{\sigma} \int_{-\infty}^{+\infty} \int_{-\infty}^{+\infty} \int_{-\infty}^{+\infty} \hat{\mathrm{f}}_{\sigma} \hat{\mathrm{v}}_{\mathrm{x}} \mathrm{d} \hat{\mathrm{v}}_{\mathbf{x}} \mathrm{d} \hat{\mathrm{v}}_{\mathbf{y}} \mathrm{d} \hat{\mathrm{v}}_{\mathrm{z}} \\
& \hat{\mathrm{u}}_{\mathbf{y}}=\frac{1}{\hat{\mathrm{n}}} \sum_{\sigma} \int_{-\infty}^{+\infty} \int_{-\infty}^{+\infty} \int_{-\infty}^{+\infty} \hat{\mathrm{f}}_{\sigma} \hat{\mathrm{v}}_{\mathbf{y}} \mathrm{d} \hat{\mathrm{v}}_{\mathbf{x}} \mathrm{d} \hat{\mathrm{v}}_{\mathbf{y}} \mathrm{d} \hat{\mathrm{v}}_{\mathrm{z}} \\
& \hat{\mathrm{u}}_{\mathrm{z}}=\frac{1}{\hat{\mathrm{n}}} \sum_{\sigma} \int_{-\infty}^{+\infty} \int_{-\infty}^{+\infty} \int_{-\infty}^{+\infty} \hat{\mathrm{f}}_{\sigma} \hat{\mathrm{v}}_{\mathbf{z}} \mathrm{d} \hat{\mathrm{v}}_{\mathbf{x}} \mathrm{d} \hat{\mathrm{v}}_{\mathbf{y}} \mathrm{d} \hat{\mathrm{v}}_{\mathbf{z}} \\
& \hat{\mathrm{T}}_{\mathrm{t}}=\frac{2}{3 \hat{\mathrm{n}}}\left[\sum _ { \sigma } \int _ { - \infty } ^ { + \infty } \int _ { - \infty } ^ { + \infty } \int _ { - \infty } ^ { + \infty } \left[\left(\hat{\mathrm{v}}_{\mathrm{x}}-\hat{\mathrm{u}}_{\mathrm{x}}\right)^{2}+\left(\hat{\mathrm{v}}_{\mathbf{y}}-\hat{\mathrm{u}}_{\mathrm{y}}\right)^{2}\right.\right. \\
& \left.+\left(\hat{v}_{z}-\hat{u}_{z}\right)^{2}\right] \hat{f}_{\sigma} d \hat{v}_{x} d \hat{v}_{y} d \hat{v}_{z} \\
& \hat{\mathrm{T}}_{\mathrm{i}}=\frac{1}{\hat{\mathrm{n}}} \sum_{\sigma} \hat{\mathrm{n}}_{\sigma} \hat{\mathrm{E}}_{\sigma} \\
& \hat{\mathrm{T}}_{\mathrm{T}}=\frac{3}{5} \hat{\mathrm{T}}_{\mathrm{t}}+\frac{2}{5} \hat{\mathrm{T}}_{\mathrm{i}}
\end{aligned}
$$




\section{Discretization of Distribution Function and Numerical Scheme}

The distribution function at energy level $\sigma, \hat{\mathrm{f}}_{\sigma}$, was discretized with equal spacing in the physical space, the arguments of the odd equally spaced quadrature (see appendix and ref. 18) was the spacing for the velocity space, and the roots of the Laguerre polynomial of degree 4 was used for the spacing for the internal energy space. The value of the distribution function at these discretized points was calculated by solving the Boltzmann equation using the finite difference method in the physical space.

The difference scheme is the first-order upwind difference based on the molecular velocity; for example,

$$
\frac{\partial \hat{\mathrm{f}}_{\sigma}}{\partial \hat{\mathrm{x}}}=\frac{\hat{\mathrm{f}}_{\sigma, \mathbf{i}, \mathbf{j}, \mathbf{k}, \alpha, \beta, \gamma}-\hat{\mathrm{f}}_{\sigma, \mathbf{i}-1, \mathbf{j}, \mathbf{k}, \alpha, \beta, \gamma}}{\Delta \hat{\mathbf{x}}} \quad \text { when } \hat{\mathbf{v}}_{\mathbf{x}} \succ 0
$$

and

$$
\frac{\partial \hat{\mathrm{f}}}{\partial \hat{\mathrm{x}}}=\frac{\hat{\mathrm{f}}_{\sigma, \mathbf{i}+1, \mathrm{j}, \mathrm{k}, \alpha, \beta, \gamma}-\hat{\mathrm{f}}_{\sigma, \mathrm{i}, \mathrm{j}, \mathrm{k}, \alpha, \beta, \gamma}}{\Delta \hat{\mathrm{x}}} \quad \text { when } \hat{\mathrm{v}}_{\mathbf{x}} \prec 0
$$

The distribution functions were then integrated for all macroscopic properties by using the odd equally spaced quadrature, e.g., the particle density at energy level $\sigma$ and at the physical point $(\mathrm{i}, \mathrm{j}, \mathrm{k})$ is

$$
\int_{-\infty}^{+\infty} \int_{-\infty}^{+\infty} \int_{-\infty}^{+\infty} \hat{\mathrm{f}}_{\sigma} d \hat{\mathrm{v}}_{\mathbf{x}} \mathrm{d} \hat{\mathrm{v}}_{\mathbf{y}} \mathrm{d} \hat{\mathrm{v}}_{\mathrm{z}} \cong \sum_{\alpha=-\mathrm{n}^{\prime}}^{\mathrm{n}^{\prime}} \sum_{\beta=-\mathbf{n}^{\prime \prime}}^{\mathrm{n}^{\prime \prime}} \sum_{\gamma=-\mathbf{n}^{\prime \prime}}^{\mathrm{n}^{\prime \prime \prime}} \mathrm{k}_{\alpha^{\prime}} \mathrm{k}_{\beta} \mathrm{k}_{\gamma} \hat{\mathrm{f}}_{\sigma}(\mathrm{i}, \mathrm{j}, \mathrm{k}, \alpha, \beta, \gamma)
$$

where $\mathrm{k}_{\alpha}, \mathrm{k}_{\beta}$, and $\mathrm{k}_{\gamma}$ are the weighting coefficients of the odd equally spaced quadrature for the velocity components $\hat{v}_{\alpha}, \hat{v}_{\beta}$, and $\hat{v}_{\gamma}$, respectively. These properties were summed over the energy space, and then saved and used for the next iteration.

\section{Boundary Conditions}

For a constantly moving surface, perfectly diffuse reflection is assumed to specify the interaction of the molecules with the surface of the moving plate. Molecules which strike the moving surface $(j=j \max$ surface $)$ are emitted with a Maxwellian velocity distribution characterized by the plate temperature $\hat{\mathrm{T}}_{\mathrm{w}}$ and the plate velocity $\hat{\mathrm{w}}$; that is,

$$
\hat{\mathrm{f}}_{\sigma}=\hat{\mathrm{n}}_{\mathrm{w}}\left(\frac{1}{\pi \hat{\mathrm{T}}_{\mathrm{w}}}\right)^{3 / 2} \mathrm{e}
$$


where the density of molecules diffusing from the plate $\hat{n}_{w}$ is not known a priori and must be found by applying the condition of zero mass flux normal to the plate at the surface, i.e.,

$$
\sum_{\sigma}\left\{\int_{-\infty}^{\infty} \int_{0}^{\infty} \int_{-\infty}^{\infty} \hat{v}_{y} \hat{f}_{\sigma}^{+} d \hat{v}_{x} d \hat{v}_{y} d \hat{v}_{z}+\int_{-\infty}^{\infty} \int_{-\infty}^{0} \int_{-\infty}^{\infty} \hat{v}_{y} \hat{f}_{\sigma}^{-} d \hat{v}_{x} d \hat{v}_{y} d \hat{v}_{z}\right\}=0
$$

where $\hat{\mathrm{f}}_{\sigma}^{+}$is the distribution function for $\hat{\mathbf{v}}_{\mathbf{y}}>0$ and $\hat{\mathrm{f}}_{\sigma}^{-}$is the distribution function for $\hat{v}_{\mathbf{y}}<0$. In the discrete ordinate form, this gives the relation at $(i, j, k)$

$$
\hat{\mathrm{n}}_{\mathrm{w}}=\frac{2(\pi)^{1 / 2}}{\hat{\mathrm{T}}_{\mathrm{w}}^{1 / 2}} \sum_{\sigma=1}^{\mathrm{n}} \sum_{\alpha=-\mathrm{n}^{\prime}}^{\mathrm{n}^{\prime}} \sum_{\beta=1}^{\mathrm{n}^{\prime \prime}} \sum_{\gamma=-\mathrm{n}^{\prime \prime \prime}}^{\mathrm{n}^{\prime \prime \prime}} \mathrm{k}_{\alpha} \mathrm{k}_{\beta} \mathrm{k}_{\gamma} \hat{\mathrm{v}}_{\beta} \hat{\mathrm{f}}_{\sigma, \alpha, \beta, \gamma}
$$

Similarly, a zero-mass-flux boundary condition was applied to all other surfaces, and a Maxwellian velocity distribution function characterized by the plate temperature was used for the molecules emitted from the surface.

Because of the symmetry of the flow inside the cubic cavity, half of the cubic cavity was used for the computation. At the symmetry plane (i.e., $\mathrm{k}=\mathrm{kmax}$ plane), the symmetry boundary condition was applied; that is, the distribution function of the outgoing molecule is equal to the distribution function of the incoming molecule at the symmetry plane.

\section{Computational Procedures}

For the present study, the initial condition assumed was that the flow everywhere was characterized by $\hat{\mathrm{n}}=1$ and $\hat{\mathrm{T}}=1$. As mentioned previously, the $\mathrm{j}=\mathrm{jmax}$ surface was selected to be the moving surface. The computation began from the $\mathrm{j}=2$ surface with a known value for $\hat{\mathrm{f}}_{\sigma}$ at the $\mathrm{j}=1$ surface. On each $\mathrm{j}=$ constant surface, the computation was performed along the $\mathrm{i}$ direction first and then marching toward the symmetry plane $(\mathrm{k}=\mathrm{kmax})$ starting with $\hat{\mathrm{f}}_{\sigma}^{+++}$. The symbol $\hat{\mathrm{f}}_{\sigma}^{+++}$is defined as the subdistribution function at energy level $\sigma$ for $\hat{\mathbf{v}}_{\mathbf{x}}>0$, $\hat{v}_{y}>0$, and $\hat{v}_{z}>0$. At the symmetry plane, the symmetry boundary condition was applied (i.e., $\hat{\mathrm{f}}^{++-}=\hat{\mathrm{f}}^{+++}$). Knowing $\hat{\mathrm{f}}_{\sigma}^{++-}$at the symmetry plane, the computation was marched backward from $k=k \max -1$ toward $\mathrm{k}=1$. Similarly, the computation continued for $\hat{\mathrm{f}}_{\sigma}^{-++}$and $\hat{\mathrm{f}}_{\sigma}^{-+-}$.

After the computation reached the $\mathrm{j}=\mathrm{jmax}$ surface, the particle density was found at this surface by applying the no-flux boundary condition and then the computation marched downward from $j=j \max -1$ to $\mathrm{j}=1$ surface. The order of computation was $\hat{\mathrm{f}}_{\sigma}^{+-+}$, $\hat{\mathrm{f}}_{\sigma}^{+--}, \hat{\mathrm{f}}_{\sigma}^{--+}$, and $\hat{\mathrm{f}}_{\sigma}^{---}$. The particle density on the surfaces could then be found by using the no-flux boundary condition and the iteration 
continued. The fourth-order Gauss-Laguerre quadrature was used for the energy space, and four sets of the third-order odd equally spaced quadrature were used for the molecular velocity space with the equal spacing of 0.15 . (See the appendix.) The convergence was assumed when the maximum particle density increment was less than $10^{-4}$.

\section{Results}

As mentioned previously, efforts have been made to calculate the flow with as small a Knudson number as possible so that the results could be compared with the solution of the Navier-Stokes equation. The results of this study are all based on $\hat{w}=0.1$. Comparisons between the Boltzmann solution and the Navier-Stokes solution ${ }^{14}$ are given in figures 2 to 4 .

Figure 2 shows the velocity profiles along the centerline for free molecule flow (Knudson nuinter $(\mathrm{Kn})=100)$, slip flow $(\mathrm{Kn}=0.1)$, and nearly continuum flow $(\mathrm{Kn}=0.03)$. It clearly shows that the flow slips on the moving surface for $K n=100$ and $K n=0.1$; that is, the flow velocity on the surface is less than the speed of the moving wall. It also shows that the no-slip boundary condition of the Navier-Stokes equation was almost recovered for $\mathrm{Kn}=0.03$.

The comparison of the velocity vector plot on the symmetry plane between the Navier-Stokes solution and the Boltzmann solution is given in figure 3. The general shapes of the primary vortex are similar except that the center of the vortex for the Navier-Stokes solution is slightly farther downstream than that for the Boltzmann solution.

Figure 4 shows the surface static pressure looking from the center of the cubic cavity toward the upper corner for both the Navier-Stokes solution and the Boltzmann solution. The three-dimensional effect of the static pressure distribution is clearly shown qualitatively. The Boltzmann solution, even for the case of $\mathrm{Kn}=0.03$, is still not close to the continuum solution obtained by the Navier-Stokes equation; however, the consistent trend toward the Navier-Stokes solution is encouraging.

The symmetry plane velocity vector plots for two different Knudson numbers are shown in figure 5 . The shape of the primary vortex is clearly shown even for $\mathrm{Kn}=100$. The center of the vortex moves upward toward the center obtained by the Navier-Stokes solution (fig. 3) when the Knudson number is reduced. It also clearly shows that the magnitude of the velocity vectors increases as expected when the Knudson number is reduced.

Figure 6 is a plot of the number particle density on the surfaces for three Knudson numbers. The distribution patterns for $K n=100$ and $\mathrm{Kn}=0.03$ are completely different. For a free molecular flow $(\mathrm{Kn}=100)$, there are not enough molecular collisions to ensure the high number particle density at the upper downstream corner as shown for the case of $\mathrm{Kn}=0.03$. The high number particle density on the upper corner of the downstream vertical surface (i.e., near $\mathrm{i}=\mathrm{imax}$ and $\mathrm{j}=\mathrm{jmax}$ ) and the low number 
particle density on the upper corner of the upstream vertical surface (i.e., near $\mathrm{i}=1$ and $\mathrm{j}=\mathrm{jmax}$ ) is a reasonable solution.

The convergence history is shown in figure 7 for $\mathrm{Kn}=0.03$. Each iteration took about $40 \mathrm{~min}$ on the Cray 2 supercomputer. Fortunately, it required less than 50 iterations to reach a convergent solution. In other words, it took less than $30 \mathrm{hr}$ of CPU time for $\mathrm{Kn}=0.03$. The CPU time for $\mathrm{Kn}=100$ was less than $10 \mathrm{hr}$. To reduce the main memory requirement, the Maxwellian distribution functions and collision frequency were calculated repeatedly; as a consequence, extra CPU time was needed for each iteration. The grid size used in the study was 31 by 31 by 16 in the physical space, 12 by 12 by 12 in the molecular velocity space and 4 levels of internal energy in the energy space. Considerable CPU time can be saved by improving the integration scheme in the molecular velocity space. In any case, the discrete ordinate method used in this study can be applied to simple three-dimensional geometries without using the parallel programming technique.

\section{Concluding Remarks}

A three-dimensional cubic cavity flow was solved for diatomic gases by using the Boltzmann equation with the Bhatnagar-Gross-Krook (B-G-K) model. A comparison was made between the Boltzmann solution and the Navier-Stokes solution for the velocity profiles along the centerline, the primary vortex on the symmetry plane, and the surface static pressure. The general trend toward the Navier-Stokes solution as the Knudson number is reduced indicates that the solutions are very reasonable and that the discrete ordinate method can be used with confidence to a three-dimensional geometry for practical predictions of rarefied-flow characteristics. Because of the robustness of this numerical scheme, it requires less than $\mathbf{5 0}$ iterations to obtain a converged solution. The present method has a potential to be a practical flow simulation method to cover all flow regimes.

\section{Appendix-Laguerre and Odd Equally Spaced Quadratures}

\section{Abscissa and Weight Factors for Laguerre Integration}

$$
\int_{0}^{\infty} e^{-E} f(E) d E=\sum_{i=1}^{n} R_{i} f\left(E_{i}\right)
$$

where $\mathrm{E}_{\mathrm{i}}$ are the abscissas and are the roots of Laguerre polynomials.

For $\mathrm{n}=4$,

$$
\mathrm{E}_{1}=0.322547689619
$$




$$
\begin{aligned}
& \mathrm{E}_{2}=1.745761101158 \\
& \mathrm{E}_{3}=4.536620296921 \\
& \mathrm{E}_{4}=9.395070912301 \\
& \mathrm{R}_{1}=6.03154104342 \times 10^{-1} \\
& \mathrm{R}_{2}=3.57418692438 \times 10^{-1} \\
& \mathrm{R}_{3}=3.88879085150 \times 10^{-2} \\
& \mathrm{R}_{4}=5.39294705561 \times 10^{-4}
\end{aligned}
$$

\section{Odd Equally Spaced Quadrature}

$$
\int_{0}^{1} \mathrm{f}(\mathrm{x}) \mathrm{dx} \cong \sum_{\mathrm{i}=1}^{\mathrm{n}} \mathrm{k}_{\mathrm{i}} \mathrm{f}\left(\alpha_{\mathrm{i}}\right)
$$

where the arguments $\alpha_{\mathrm{i}}$ are taken to be $1 /(2 \mathrm{n}), 3 /(2 \mathrm{n}), \ldots, 1-[1 /(2 \mathrm{n})]$ and the weighting coefficients $k_{i}$ for $n=3$ are

$$
\begin{aligned}
& \mathrm{k}_{1}=\mathrm{k}_{3}=0.375 \\
& \mathrm{k}_{2}=0.25
\end{aligned}
$$

References
${ }^{1}$ Huang, A.B. and Hwang, D.P., "Test of Statistical Models for Gases With and Without Internal Energy States," Physics of Fluids, Vol. 16, No. 4, 1973, pp. 466-475.

${ }^{2}$ Huang, A.B., Hwang, D.P., Giddens, D.P., and Srinivasan, R., "HighSpeed Leading Edge Problem," Physics of Fluids, Vol. 16, No 6, 1973, pp. 814-824.

${ }^{3}$ Dogra, V.K. and Moss, J.N., "Hypersonic Rarefied Flow About Plates at Incidence," AIAA Paper 89-1712, 1989.

${ }^{4}$ Chung, C., DeWitt, K., Jeng, D., and Keith, T., "Rarefied Gas Flow Through Two-Dimensional Nozzles," AIAA Paper 89-2893, 1989.

${ }^{5}$ Chung, C.H., Keith, T.G., Jeng, D.R., and DeWitt, K.J., "Numerical Simulation of Rarefied Gas Flow Through a Slit," Journal of Thermophysics and Heat Transfer, Vol. 6, No. 1, 1992, pp. 27-34.

${ }^{6}$ Koura, K., "Direct Simulation of Vortex Shedding in Dilute Gas Flows Past an Inclined Flat Plate," Physics of Fluids, A, Vol. 2, No. 2, 1990, pp. 209-213.

${ }^{7}$ Chung, C.H., DeWitt, K.J, and Jeng, D.R., "New Approach in DirectSimulation of Gas Mixtures," AIAA Paper 91-1343, 1991.

${ }^{8}$ Lin, T.C., McGregor, R.D., Wong, J.L., and Grabowsky, W.R., "Numerical Simulation of 3D Rarefied Hypersonic Flows," AIAA Paper 89-1715, 1989.

${ }^{9}$ Sreekanth, A.K. and Davis, A., "Rarefied Gas Flow Through Rectangular Tubes: Experimental and Numerical Investigation," Rarefied Gas Dynamics: Space-related Studies, International Symposium, 
E.P. Muntz, D.P. Weaver, and D.M. Campbell, eds., AIAA, Washington, DC, 1989, pp. 257-272.

${ }^{10}$ Celenligil, M. and Moss, J., "Direct Simulation of Hypersonic Rarefied Flow About a Delta Wing," AIAA Paper 90-0143, 1990.

${ }^{11}$ Huang, A.B., Report No. 4, "A General Discrete Ordinate Method for the Dynamics of Rarefied Gases," Georgia Institute of Technology, School of Aerospace Engineering Rarefied Gas Dynamics and Plasma Laboratory, 1967.

${ }^{12}$ Bhatnagar, P.L., Gross, E.P., and Krook, M., "A Model for Collision Processes in Gases," I. Small Amplitude Processes in Charged and Neutral One-Component Systems," Physical Review, Vol. 94, No. 3, 1954, pp. 511-525.

${ }^{13}$ Huang, A.B., "Nonlinear Rarefied Couette Flow with Heat Transfer," Physics of Fluids, Vol. 11, No. 6, 1968, pp. 1321-1326.

${ }^{14}$ Hwang, D.P. and Huynh, T.H., "A Finite Difference Scheme for ThreeDimensional Steady Laminar Incompressible Flow," NASA TM-89851, 1987.

${ }^{15}$ Morse, T.F., "Kinetic Model for Gases with Internal Degrees of Freedom," Physics of Fluids, Vol. 7, No. 2, 1964, pp. 159-169.

${ }^{16}$ Holway, L.H., Jr., "New Statistical Models for Kinetic Theory: Methods of Construction," Physics of Fluids, Vol. 9, No. 9, 1966, pp. 1658-1673.

${ }^{17}$ Chapman, S. and Cowling, T.G., The Mathematical Theory of NonUniform Gases: An Account of the Kinetic Theory of Viscosity, Thermal Condition, and Diffusion in Gasses, Cambridge University Press, London, UK, 1958, p. 223.

${ }^{18}$ Hwang, D.P., "A Kinetic Theory Description of Rarefied Gas Flows with the Effect of Rotational Relaxation," Ph.D. Thesis, Georgia Institute of Technology, Atlanta, GA, 1971.

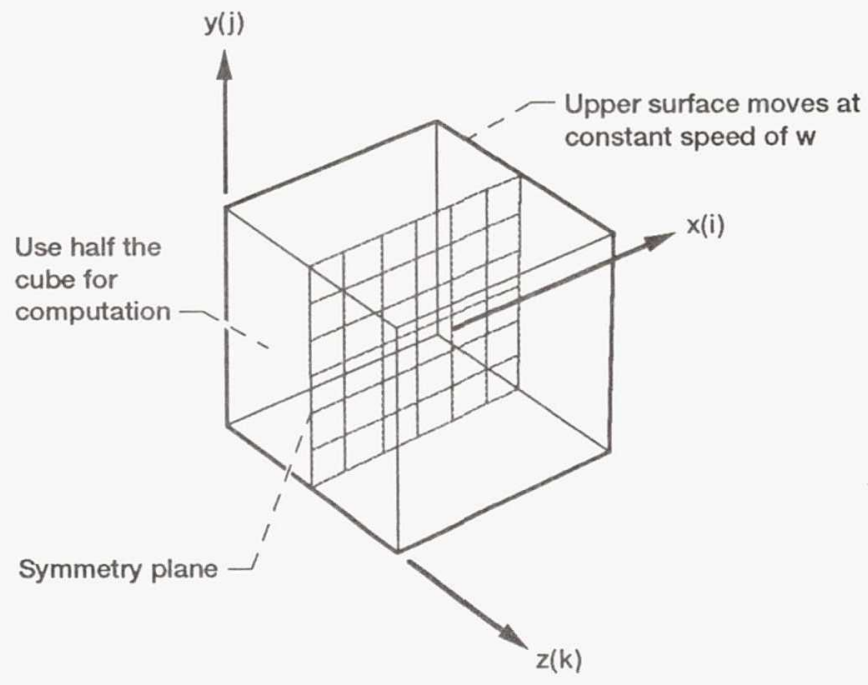

Figure 1.-Geometry. 


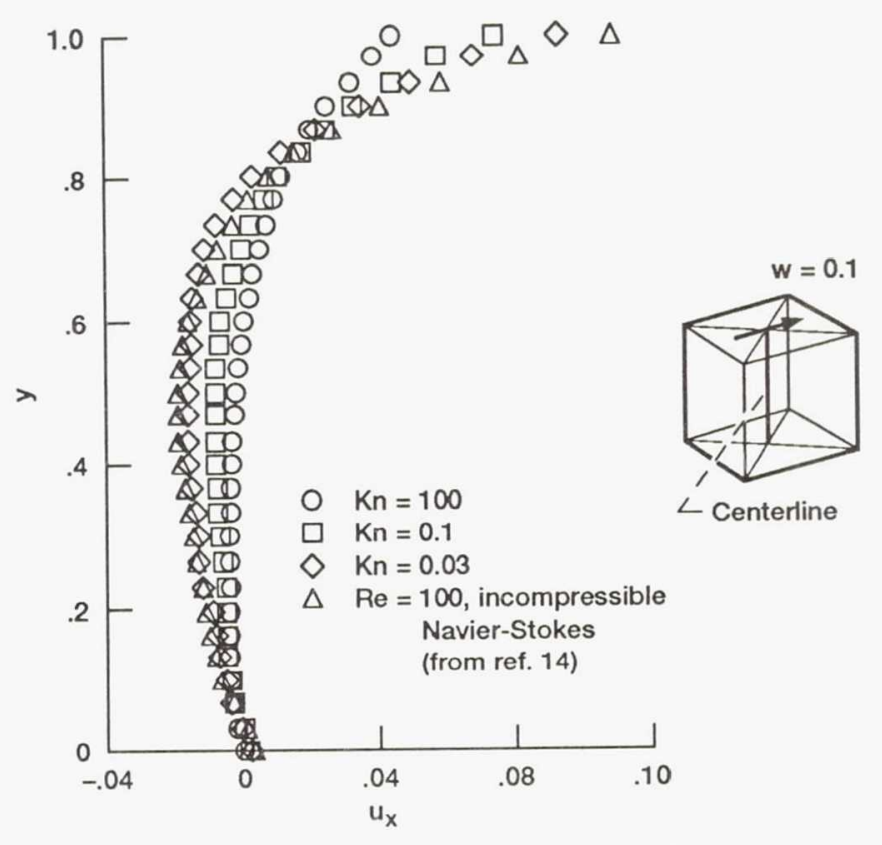

Figure 2.-Velocity profiles along centerline.

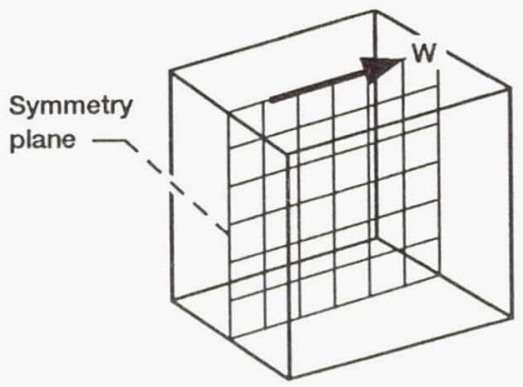

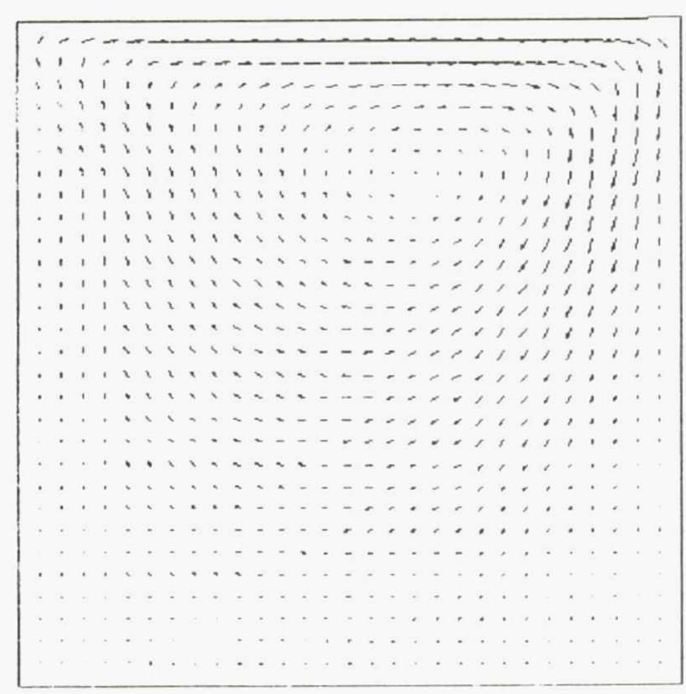

(a) Navier-Stokes solution ( $R e=100)$.

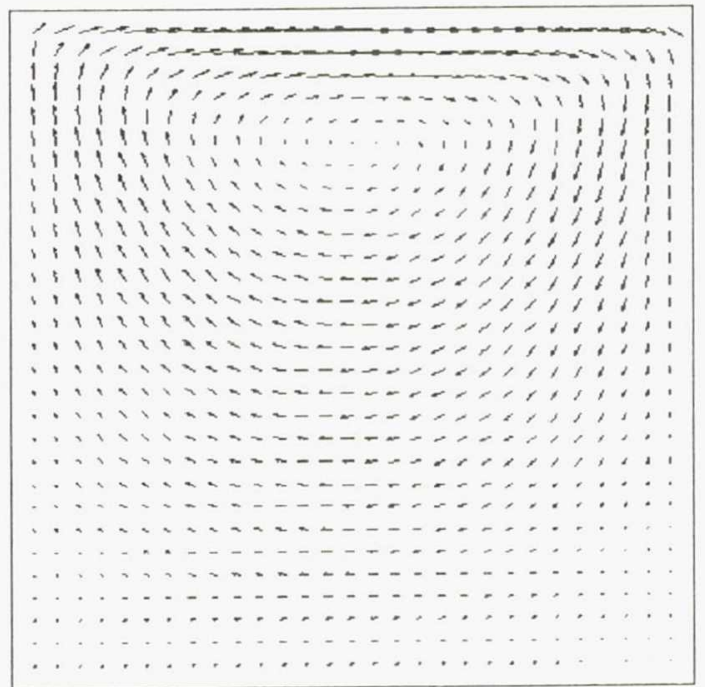

(b) Boltzmann solution $(K n=0.03)$

Figure 3.-Velocity vectors on symmetry plane for Navier-Stokes solution and Boltzmann solution. 
Page intentionally left blank 


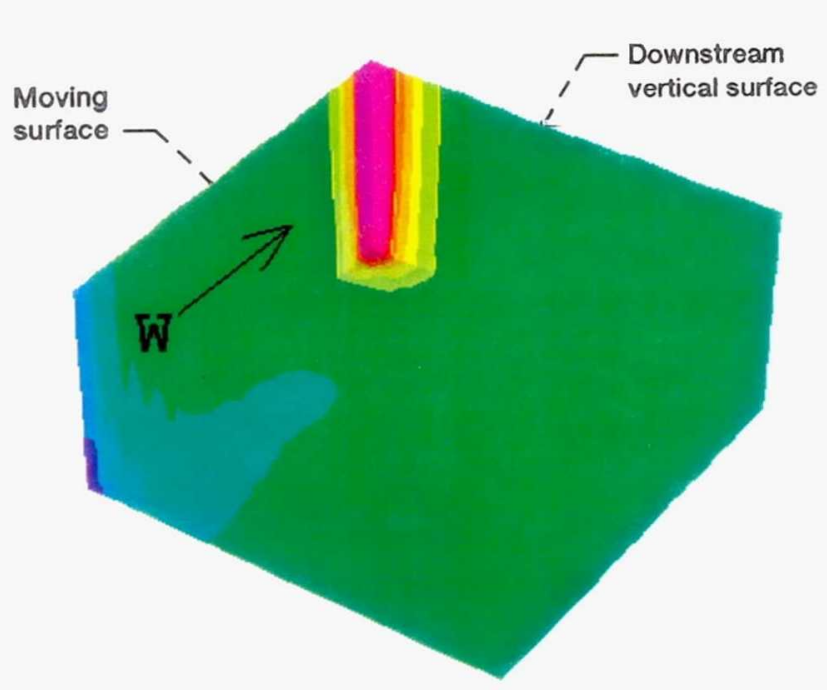

(a) Navier-Stokes solution $(\mathrm{Re}=100)$.

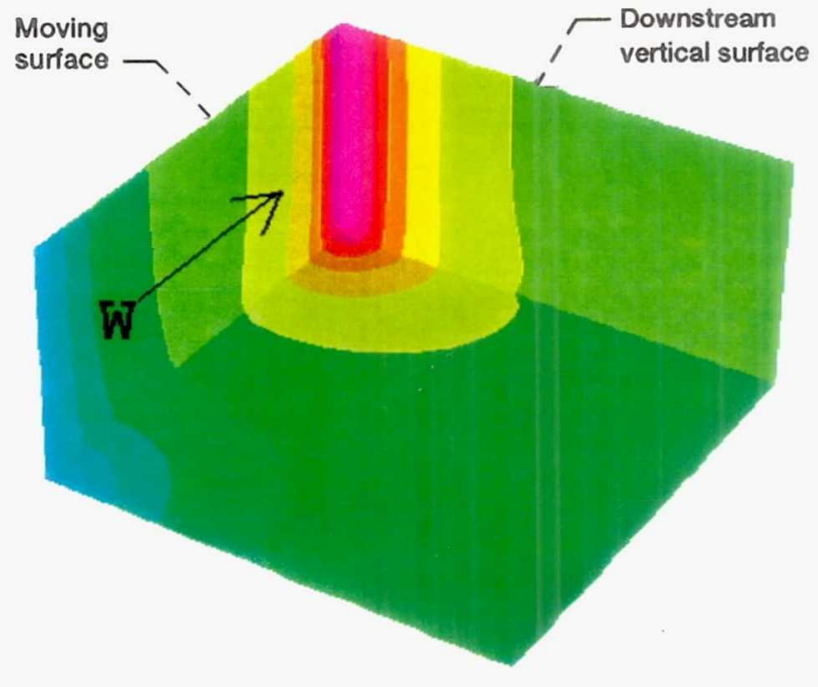

(b) Boltzmann solution ( $K n=0.03$ ).

Figure 4.- Surface static pressure for Navier-Stokes solution and Boltzmann solution.

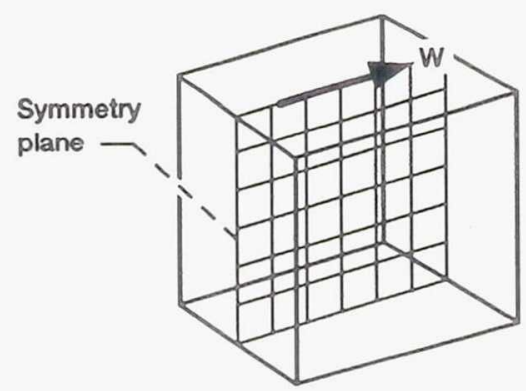

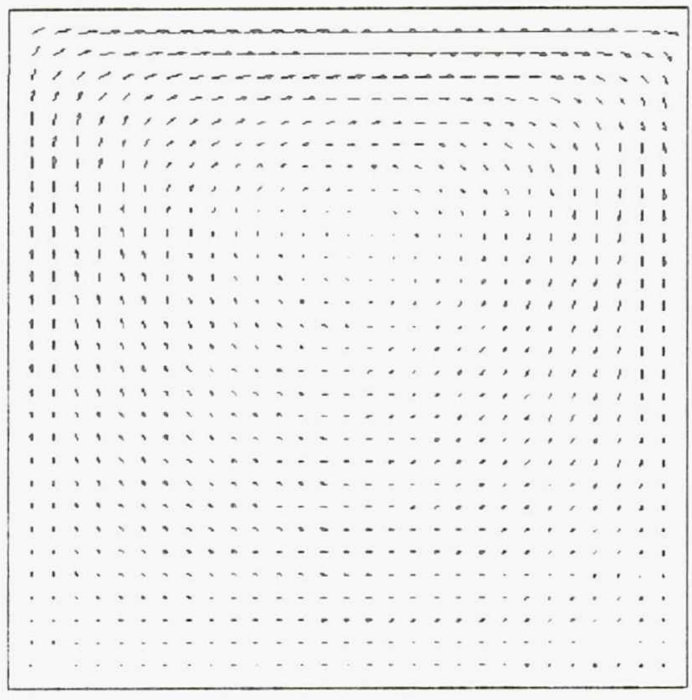

(a) $\mathrm{Kn}=100$.

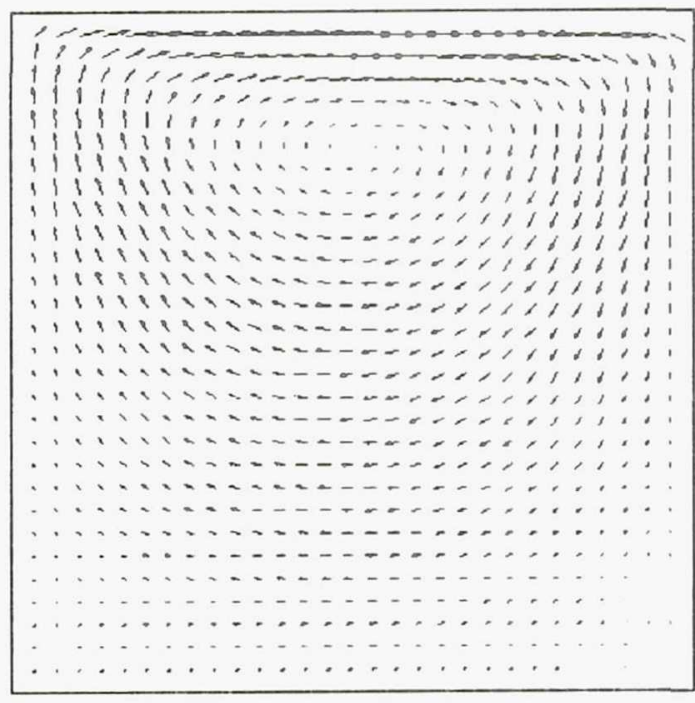

(b) $\mathrm{Kn}=0.03$

Figure 5.-Velocity vectors on symmetry plane for two Knudson numbers. 
Page intentionally left blank 


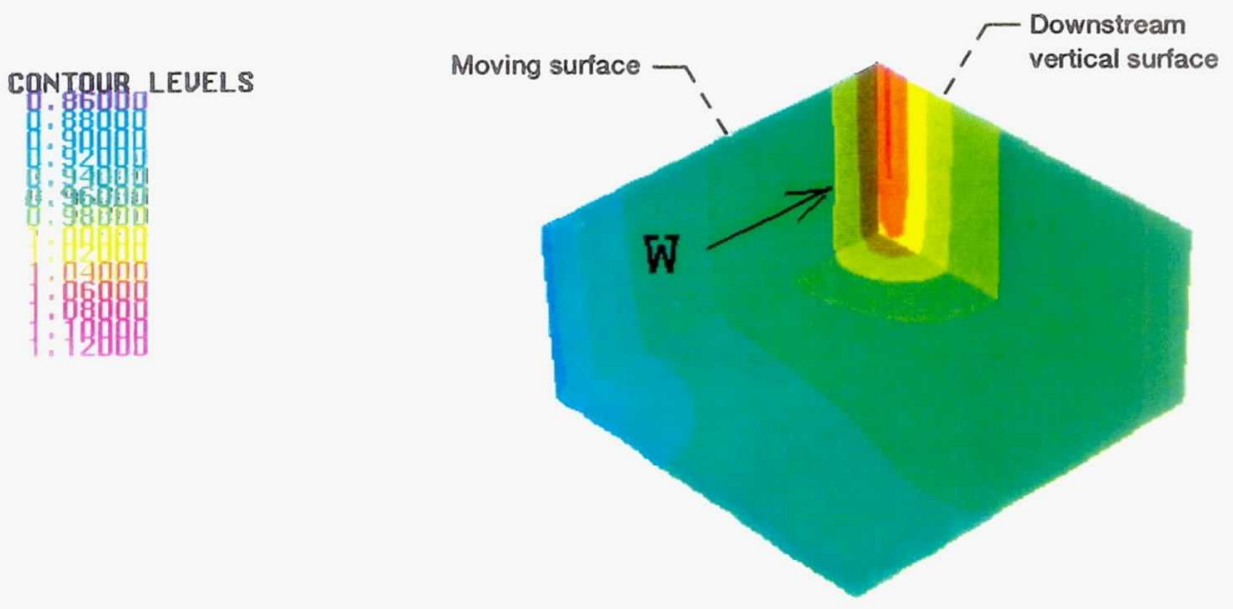

(a) $K n=0.1$.

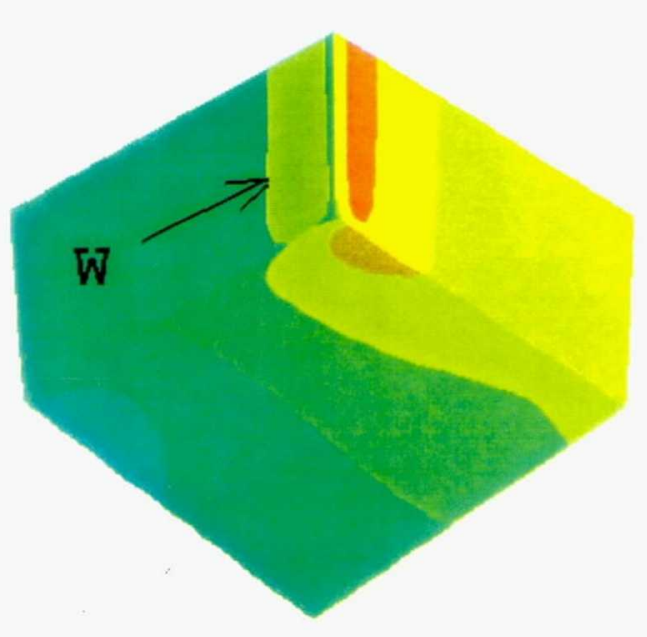

(b) $K n=100$.

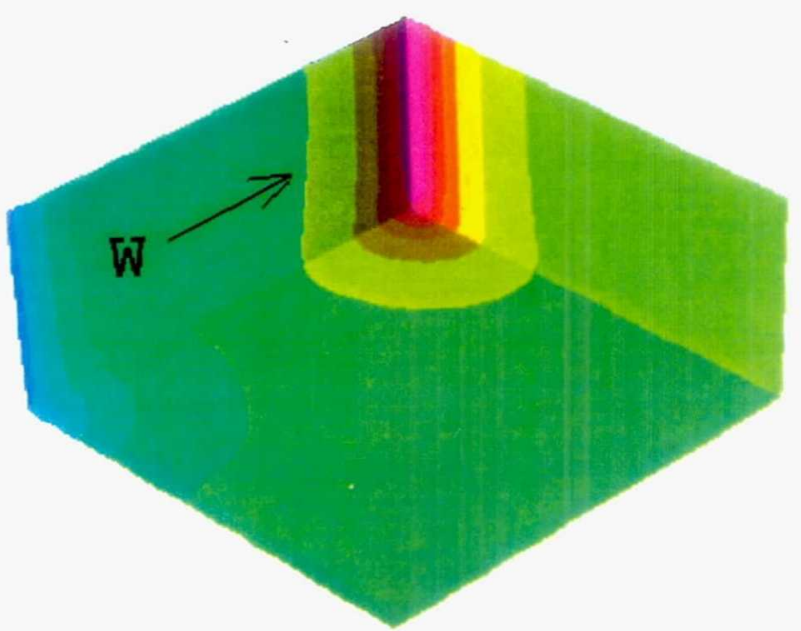

(c) $\mathrm{Kn}=0.03$.

Figure 6.-Number particle density on surfaces for three Knudson numbers. 
Page intentionally left blank 


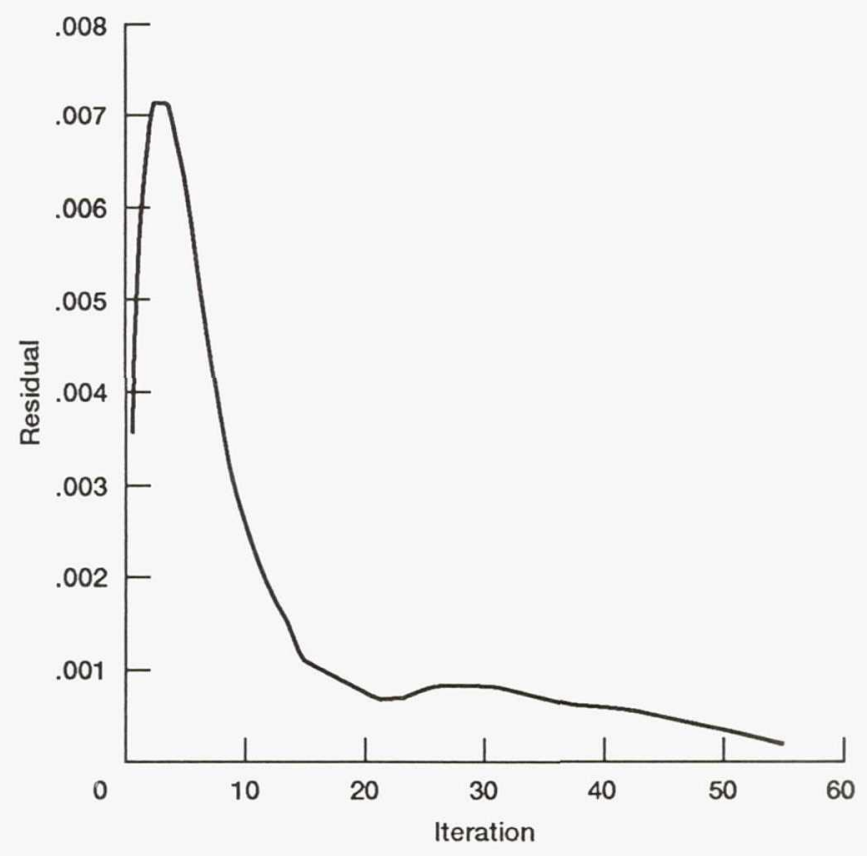

Figure 7.-Convergence history for $\mathrm{Kn}=0.03$. 
Public reporting burden for this collection of information is estimated to average 1 hour per response, including the time for reviewing instructions, searching existing data sources, gathering and maintaining the data needed, and completing and reviewing the collection of information. Send comments regarding this burden estimate or any other aspect of this collection of information, including suggestions for reducing this burden, to Washington Headquarters Services, Directorate for information Operations and Reports, 1215 Jefferson Davis Highway, Suite 1204, Arlington, VA 22202-4302, and to the Office of Management and Budget, Paperwork Reduction Project (0704-0188), Washington, DC 20503.

\begin{tabular}{|l|c|c|}
\hline 1. AGENCY USE ONLY (Leave blank) & $\begin{array}{c}\text { 2. REPORT DATE } \\
\text { July } 1992\end{array}$ & $\begin{array}{r}\text { 3. REPORT TYPE AND DATES COVERED } \\
\text { Technical Memorandum }\end{array}$ \\
\hline
\end{tabular}

\section{TITLE AND SUBTITLE}

Numerical Solution of a Three-Dimensional Cubic Cavity Flow by Using the Boltzmann Equation

\section{AUTHOR(S)}

Danny P. Hwang

7. PERFORMING ORGANIZATION NAME(S) AND ADDRESS(ES

National Aeronautics and Space Administration

Lewis Research Center

Cleveland, Ohio 44135-3191

\section{SPONSORING/MONITORING AGENCY NAMES(S) AND ADDRESS(ES)}

National Aeronautics and Space Administration

Washington, D.C. 20546-0001

\section{FUNDING NUMBERS}

WU-505-62-20

8. PERFORMING ORGANIZATION REPORT NUMBER

E-7080

10. SPONSORING/MONITORING AGENCY REPORT NUMBER

NASA TM-105693

\section{SUPPLEMENTARY NOTES}

Prepared for the 18th International Symposium on Rarefield Gas Dynamics, sponsored by the University of British Columbia, Vancouver, Canada, July 26-31, 1992. Responsible person, Danny P. Hwang, (216) 433-2187.

12a. DISTRIBUTION/AVAILABILITY STATEMENT

Unclassified - Unlimited

Subject Category 34 12b. DISTRIBUTION CODE

\section{ABSTRACT (Maximum 200 words)}

A three-dimensional cubic cavity flow has been analyzed for diatomic gases by using the Boltzmann equation with the Bhatnagar-Gross-Krook (B-G-K) model. The method of discrete ordinate was applied, and the diffuse reflection boundary condition was assumed. The results, which show a consistent trend toward the Navier-Stokes solution as the Knudson number is reduced, give us confidence to apply the method to a three-dimensional geometry for practical predictions of rarefied-flow characteristics. The CPU time and the main memory required for a three-dimensional geometry using this method seem reasonable.

\section{SUBJECT TERMS}

Boltzmann equation; Cubic cavity flow; Kinetic theory; B-G-K model, Discrete ordinate method

17. SECURITY CLASSIFICATION OF REPORT

Unclassified
18. SECURITY CLASSIFICATION OF THIS PAGE Unclassified
19. SECURITY CLASSIFICATION OF ABSTRACT Unclassified
15. NUMBER OF PAGES 20

16. PRICE CODE

$\mathrm{A03}$

20. LIMITATION OF ABSTRACT 